\title{
Development of a gene doping detection method to detect overexpressed human follistatin using an adenovirus vector in mice
}

\author{
Koki Yanazawa $^{1}$, Takehito Sugasawa $^{2}$, Kai Aoki $^{2}$, Takuro Nakano ${ }^{1}$, Yasushi Kawakami ${ }^{2}$, Kazuhiro Takekoshi ${ }^{\text {Corresp. }}$ \\ ${ }^{1}$ Graduate School of Comprehensive Human Sciences, University of Tsukuba, Tsukuba, Japan \\ 2 Laboratory of Clinical Examination/Sports Medicine, Division of Clinical Medicines, Faculty of Medicine, University of Tsukuba, Tsukuba, Japan \\ Corresponding Author: Kazuhiro Takekoshi \\ Email address: k-takemd@md.tsukuba.ac.jp
}

Background. Gene doping is the misuse of genome editing and gene therapy technologies for the purpose of manipulating specific genes or gene functions in order to improve athletic performance. However, a non-invasive detection method for gene doping using recombinant adenoviral (rAdV) vectors containing human follistatin ( $h F S T)$ genes $(r A d V<h F S T>$ ) has not yet been developed. Therefore, the aim of this study was to develop a method to detect gene doping using $\mathrm{rAdV}<h F S T>$.

Methods. First, we generated rAdV $<h F S T>$ and evaluated the overexpression of the $h F S T$ gene, FST protein, and muscle protein synthesis signaling using cell lines. Next, $r A d V<h F S T>$ was injected intravenously or intramuscularly into mice, and whole blood was collected, and hFST and cytomegalovirus promoter (CMVp) gene fragments were detected using TaqMan- quantitative polymerase chain reaction ( $\mathrm{PPCR}$ ). Finally, to confirm the specificity of the primers and the TaqMan probes, samples from each experiment were pooled, amplified using TaqMan-qPCR, and sequenced using the Sanger sequencing.

Results. The expression of hFST and FST proteins and muscle protein synthesis signaling significantly increased in $\mathrm{C} 2 \mathrm{C} 12$ cells. In long-term, transgene fragments could be detected until 4 days after intravenous injection and 3 days after intramuscular injection. Finally, the Sanger sequencing confirmed that the primers and TaqMan probe specifically amplified the gene sequence of interest.

Conclusions. These results indicate the possibility of detecting gene doping using $\mathrm{rAdV}<h F S T>$ using TaqMan-qPCR in blood samples. This study may contribute to the development of detection methods for gene doping using rAdV $<h F S T>$. 


\section{Development of a gene doping detection method to detect}

\section{2 overexpressed human follistatin using an adenovirus vector}

\section{3 in mice}

4

5 Koki Yanazawa ${ }^{1}$, Takehito Sugasawa ${ }^{2}$, Kai Aoki², Takuro Nakano ${ }^{1}$, Yasushi Kawakami ${ }^{2}$ and

6 Kazuhiro Takekoshi ${ }^{2 *}$

7

$8{ }^{1}$ Graduate School of Comprehensive Human Sciences, University of Tsukuba, 1-1-1 Tennoudai, 9 Tsukuba 305-8574, Japan

${ }^{2}$ Laboratory of Clinical Examination/Sports Medicine, Division of Clinical Medicines, Faculty of Medicine, University of Tsukuba, 1-1-1 Tennoudai, Tsukuba 305-8574, Japan

Corresponding Author: Kazuhiro Takekoshi

1-1-1 Tennoudai, Ibaraki 305-8575, Japan

Email address: k-takemed@md.tsukuba.ac.jp

\section{Abstract}

Background. Gene doping is the misuse of genome editing and gene therapy technologies for the purpose of manipulating specific genes or gene functions in order to improve athletic performance. However, a non-invasive detection method for gene doping using recombinant adenoviral (rAdV) vectors containing human follistatin $(h F S T)$ genes (rAdV $<h F S T>$ ) has not yet been developed. Therefore, the aim of this study was to develop a method to detect gene doping using $\mathrm{rAdV}<h F S T>$.

Methods. First, we generated $\mathrm{rAdV}<h F S T>$ and evaluated the overexpression of the $h F S T$ gene, FST protein, and muscle protein synthesis signaling using cell lines. Next, $\mathrm{AddV}<h F S T>$ was injected intravenously or intramuscularly into mice, and whole blood was collected, and $h F S T$ and cytomegalovirus promoter $(C M V p)$ gene fragments were detected using TaqManquantitative polymerase chain reaction (qPCR). Finally, to confirm the specificity of the primers 
29 and the TaqMan probes, samples from each experiment were pooled, amplified using TaqMan30 qPCR, and sequenced using the Sanger sequencing.

31 Results. The expression of hFST and FST proteins and muscle protein synthesis signaling 32 significantly increased in $\mathrm{C} 2 \mathrm{C} 12$ cells. In long-term, transgene fragments could be detected until 334 days after intravenous injection and 3 days after intramuscular injection. Finally, the Sanger 34 sequencing confirmed that the primers and TaqMan probe specifically amplified the gene 35 sequence of interest.

36 Conclusions. These results indicate the possibility of detecting gene doping using $\mathrm{rAdV}<h F S T>$ 37 using TaqMan-qPCR in blood samples. This study may contribute to the development of 38 detection methods for gene doping using $\mathrm{rAdV}<h F S T>$.

\section{Introduction}

42 Gene therapy using gene editing technology is emerging as a new treatment strategy for 43 various genetic and acquired diseases (Wang et al., 2019). Gene therapy drugs, such as 44 Collategene ${ }^{\circledR} \square$ (AnGes. Inc, Osaka, Japan) (PMDA, 2019), Zolgensma ${ }^{\circledR} \square$ (AveXis. Inc, IL, 45 46 USA) (Press Release Novartis, 2019), and Kymriah ${ }^{\circledR} \square$ (Novartis. Inc., Basel, Switzerland) (Press Release Novartis, 2018), have been approved. Animal experiments and clinical trials are 4 being conducted to develop gene therapy strategies against diseases such as Duchenne muscular dystrophy (Duan, 2018) and hemophilia B (High \& Anguela, 2016; Naso et al., 2017). Thus, gene therapy is progressing rapidly and is expected to become common. WADA defines gene doping as "The use of nucleic acids or nucleic acid analogues that may alter genome sequences and/ or alter gene expression by any mechanism. This includes but is not limited to gene editing, gene silencing and gene transfer technologies. " (WADA, 2021). With the rapid development of genetic engineering and gene therapy, the World AntiDoping Agency (WADA) has strongly warned against gene doping. WADA has added gene doping to the prohibited list in 2003 and established a committee in 2004 to investigate the latest advances in the field of gene therapy and methods of detecting gene doping (WADA, 2015). In January 2021, WADA has published laboratory guidelines for gene doping detection based on polymerase chain reaction (PCR) indicating the establishment of standardization methods is on its way (WADA, 2021). 
60

61

62

63

64

65

66

67

68

69

70

71

72

73

74

75

76

77

78

79

80

81

82

83

84

85

86

87

88 Materials \& Methods

89

In gene therapy, gene carriers called vectors are used. The vector data in Table 1 was taken from the Gene Therapy Clinical Trials Worldwide (Gene Therapy Clinical Trials (2021)) website. Viral vectors are widely used, with recombinant adenovirus (rAdV) vectors being the most common and in use (Liang, 2018; Xia et al., 2018; Zhang et al., 2018). Moreover, they are safe because the possibility of integrating into the human genome is very low, and they are characterized by transient increases in the expression of the target gene, followed by rapid disappearance from the body (Wold and Toth, 2013; Wold and Toth, 2017). These features are favorable for athletes who intend to engage in gene doping. Hence, we focused on the rAdV vector, which can be misused for gene doping.

One gene that can be exploited for gene doping is the human follistatin gene (hFST). In humans, FST is produced in the liver and secreted into the bloodstream by stimuli such as exercise (Hansen and Plomgaard, 2016). The secreted FST is delivered to skeletal muscles throughout the body where it binds to activin A and myostatin, inhibiting their action and suppressing TGF- $\beta$ signaling (DePaolo et al., 1997; Ketan, 1998). As a result, it alleviates Smad3-dependent suppression of Akt/mTOR/p70S6K signaling and induces skeletal muscle hypertrophy (Bodine et al., 2001). FST is on the WADA prohibited list (WADA, 2020), and there are concerns that it may be misused for gene doping.

To detect gene doping, it is necessary to detect fragments of the transgene or vector. In a previous study, quantitative polymerase chain reaction (qPCR) was shown to be the easiest and the most specific method for detecting gene fragments (Sugasawa et al., 2019). Therefore, in this study, we developed a qPCR detection method. qPCR mainly uses SYBR-Green or TaqMan polymerases for amplification. A previous study reported that TaqMan-qPCR has higher sensitivity and specificity than SYBR-Green-qPCR (Aoki et al., 2020). Therefore, we developed a detection method using TaqMan-qPCR.

Therefore, the purpose of this study was to develop a method using TaqMan-qPCR to detect gene doping of human FST gene using the rAdV vector. 
90 The following plasmids were used in this study: Gen EZ ${ }^{\mathrm{TM}}$ ORF clone hFST in pcDNA3.1(+)

91 (GenScript, NJ, USA), pENTR4 (Thermo Fisher Scientific, MA, USA), and pAd/CMV/V5-

92 DEST (Thermo Fisher Scientific). HEK 293A cells (Thermo Fisher Scientific) were used to 93 clone and amplify rAdV vectors. Gen EZ ${ }^{\mathrm{TM}}$ ORF clone FST in pcDNA3.1(+) was used as a 94 template to amplify the hFST gene with 5'-EcoRI and 3'-BamHI restriction sites using PCR. PCR 95 product was inserted into the pENTR4 plasmid using T4 ligase (Promega, 9692 Madison, WI, USA). The sequences of inserted hFST genes in the pENTR4 plasmids were 97 read using sanger sequencing and confirmed to be correct sequences. The following experiments 98 were performed in accordance with a previous study (Sugasawa et al., 2019).

99

100

101

102

103

104

105

106

107

108

109

110

111

112

113

114

115

116

117

118

119

\section{Cell experiments}

\subsection{Cell Culture}

HuH7 cells (RIKEN Bio Resource Center, Tsukuba, Japan) were cultured in Dulbecco's modified Eagle's medium (DMEM) (Thermo Fisher Scientific) containing 10\% fetal bovine serum (Wako, Osaka, Japan) and 1\% penicillin/streptomycin (Wako), in an incubator maintained at $37{ }^{\circ} \mathrm{C}$ with $5 \% \mathrm{CO}_{2}$ gas. HuH7 cell line established from a highly differentiated human hepatocellular carcinoma. HuH7 cells were used to mimic the high expression of FST in the liver. Cells were seeded in 12-well plates at a density of $1.0 \times 10^{5}$ cells and 6-well plates at a density of $2.5 \times 10^{5}$ cells. After the cells reached $90 \%$ confluence, the medium was changed to serum-free DMEM, and the cells were infected by rAdV.

C2C12 cells (RIKEN Bio Resource Center, Tsukuba, Japan) were cultured under the same conditions. $\mathrm{C} 2 \mathrm{C} 12$ is an immortalized mouse myoblast cell line.C2C12 cells were used to mimic the high expression of FST in the local muscle. After the cells reached $70-80 \%$ confluence, the medium was replaced with DMEM containing $2 \%$ horse serum and $1 \%$ penicillin/streptomycin (differentiation medium). Differentiation into myotubes was induced by culturing in the differentiation medium (changed every 2 days) for 7 days. After incubation, the medium was changed to serum-free DMEM, and the cells were infected by rAdV.

\section{2. rAdV infection}


$120 \mathrm{HuH7}$ and C2C12 seeded in 12-well plates (for total RNA/DNA extraction) were infected with

$1211.0 \times 10^{10} \mathrm{vp}$, and those seeded in 6-well plates (for total protein extraction) were infected with

$1224.0 \times 10^{10} \mathrm{vp}$ of $\mathrm{rAdV}<h F S T>$. A group with the same amount of phosphate-buffered saline

123 (PBS) and a group infected with the same amount of $\mathrm{rAdV}<m$ Cherry $>$ were considered as

124 controls. Total RNA/DNA extraction and total protein extraction were performed 4 days after

125 infection in $\mathrm{HuH} 7$ and 3 days in $\mathrm{C} 2 \mathrm{C} 12$ cells.

126

127

\subsection{Total RNA/DNA extraction}

128 Total RNA was extracted from culture cells. Sepasol RNA I Super G (Nacalai Tesque) was used 129 for the extraction, as per the manufacturer's protocol. After extraction, the concentration of RNA 130 in each sample was estimated using a microspectrophotometer (NanoDrop 1000; Thermo Fisher 131 Scientific), and $500 \mathrm{ng}$ of total RNA from each sample was reverse transcribed using $5 \times$

132 PrimeScript RT Master Mix (Takara Bio) to cDNA. The cDNA was diluted with a 4-fold amount 133 of purified water.

134 Total DNA was extracted from cell culture samples. A phenol/chloroform/isoamyl alcohol 135 solution (Nacalai Tesque) was used for extraction, as per the manufacturer's protocol. After 136 extraction, total DNA concentration of each sample was adjusted to $10 \mathrm{ng} / \mu \mathrm{L}$.

137 The synthesized cDNA was used to measure transgene expression, and total DNA was used to 138 detect the transgene fragments using SYBR-Green-qPCR.

139

140 2.4. Immunoblotting

141 Total proteins were extracted from the culture cells using lysis buffer ( $1 \%$ NP-40, 0.1\% SDS, 20

$142 \mathrm{mM}$ Tris- $\mathrm{HCl}[\mathrm{pH} 8.0], 5 \mathrm{mM}$ ethylenediamine tetraacetic acid [EDTA], $150 \mathrm{mM} \mathrm{NaCl}$, and 143 proteinase inhibitor [Nacalai Tesque]). Lysates were centrifuged at 12,000 x g for $15 \mathrm{~min}$ at 4

$144{ }^{\circ} \mathrm{C}$. Total protein concentration for each sample was measured using the BCA protein assay kit

145 (Takara Bio), and $3 \mu \mathrm{g} /$ lane $(\mathrm{HuH7})$ or $10 \mu \mathrm{g} /$ lane $(\mathrm{C} 2 \mathrm{C} 12)$ of total protein was used for gradient 146 gel electrophoresis. For western blotting, the blots were incubated with primary antibodies. Table 147 S1 lists the antibodies used in this study. Horseradish peroxidase-conjugated anti-rabbit IgG and 148 anti-mouse IgG were used as the secondary antibodies. Signals were detected using a 149 chemiluminescent reagent (EzWestLumi One; ATTO, Tokyo, Japan). Blots were scanned using 
150 a Light-Capture Cooled CCD Camera System (Image Quant LAS-4000; GE Healthcare,

151 Chicago, IL, USA).

152

153

2.5. Immunofluorescence

154 Immunofluorescence was performed 3 days after $\mathrm{rAdV}<h F S T>$ infection of $\mathrm{C} 2 \mathrm{C} 12$ cells. After

155 removing the supernatant, cells were fixed with 4\% paraformaldehyde (Wako) for 15 min at 20

$156{ }^{\circ} \mathrm{C}$. Subsequently, the cells were permeabilized using 0.1\% TritonX-100 (Nacalai Tesque) in

157 PBS and blocked with 5\% goat serum (Sigma-Aldrich, MO, USA) in PBS for $1 \mathrm{~h}$ at $20{ }^{\circ} \mathrm{C}$. The

158 primary antibody against Follistatin (Proteintech Group, Chicago, IL, USA, 60060-6-lg, 1:300)

159 was diluted in 5\% goat serum in PBS and incubated with the samples for $1 \mathrm{~h}$ at $20{ }^{\circ} \mathrm{C}$. Wells

160 were washed with PBS three times and incubated with the secondary antibody (Alexa Fluor 488

161 anti-mouse IgG, Jackson ImmunoResearch Laboratories, Inc., PA, USA, 1:400) for $1 \mathrm{~h}$ at $20^{\circ} \mathrm{C}$.

162 Finally, the wells were washed with PBS three times, stained with Dapi-Fluoromount-G ${ }^{\circledR}$

163 (SouthernBiotech, AL, USA), and subjected to fluorescence microscopy.

164

165 3. Animal Experiments

166 3.1. Animal

167 The animal experiments conducted in this study were approved by the Animal Experiment

168 Committee of the University of Tsukuba (Approval Number: 20-378). Six-week-old male ICR

169 mice were purchased from Central Laboratories for Experimental Animals (Tokyo, Japan) and

170 subjected to a one-week acclimation period in a cage (Maximum of 5 mice in one cage). Mice

171 were housed in an air-conditioned, pathogen-free animal room with a 12/12 h light/dark cycle.

172 The mice were allowed to consume normal solid food and water ad libitum. At the beginning of

173 the experiment, the mice weighed 34.1 37.4 $\mathrm{g}$ and were 7 weeks old. If weight loss was less than

$17420 \%$ during the experiment, or if there was obvious injury or illness, euthanasia was to be

175 performed. There were no mice that corresponded to the above in this experiment. The sample

176 size for each experiment was determined with previous study (Sugasawa et al., 2019).

177

178 3.2. Development of a detection method for gene doping using $\operatorname{rAdV}<h F S T>$

179 3.2.1. Long-term detection of transgene fragments for intravenous injection 
180 Approximately, $50 \mu \mathrm{L}$ of whole blood was collected from the tail vein of 10 untreated mice aged

1817 weeks into a $1.5-\mathrm{mL}$ tube containing $150 \mu \mathrm{L}$ of EDTA-disodium salt (EDTA-2Na)/PBS

182 mixture, which was used as a pre-injection sample. Four days later, $2.0 \times 10^{11} \mathrm{vp}$ of

$183 \mathrm{rAdV}<h F S T>$ was injected through the orbital venous plexus. Thereafter, approximately $50 \mu \mathrm{L}$

184 of whole blood was collected from the tail vein at 3, 6, and $12 \mathrm{~h}$, and 1, 2, 3, 4, 5, 6, and 7 days

185 after injection.

186 Total DNA was extracted using a phenol/chloroform/isoamyl alcohol solution (Nacalai tesque). $\square$ After

187 extraction, the concentration of DNA each sample was adjusted to $10 \mathrm{ng} / \mu \mathrm{L}$, and DNA was

188 subjected to TaqMan qPCR to detect $h F S T$ and CMVp gene fragments.

189

190 3.2.2. Detection of transgene fragments in each specimen for intravenous injection

191 Fourteen untreated mice aged 7 weeks were randomly divided into two groups: group 1 (G1) ( $n$ $192=6)$ and group $2(\mathrm{G} 2)(n=8)$. G1 was injected with $100 \mu \mathrm{L}$ of PBS, and G2 was injected with $193100 \mu \mathrm{L}$ of $2.0 \times 10^{12} \mathrm{vp} / \mathrm{mL} \mathrm{rAdV}<h F S T>$ through the orbital venous plexus. Six hours after 194 injection, the mice were administered general anesthesia by isoflurane inhalation, followed by 195 whole blood collection with EDTA-2Na. The mice were euthanized by cervical dislocation.

196 Whole blood was centrifuged at $5000 \mathrm{rpm}$ for $10 \mathrm{~min}$ at $4{ }^{\circ} \mathrm{C}$, and plasma and blood cell 197 fractions were collected separately. Each sample was stored at $-20^{\circ} \mathrm{C}$ until the next analysis. 198 Total DNA was extracted from each sample using NucleoSpin ${ }^{\circledR}$ cfDNA XS (Macherey-Nagel) 199 for plasma fractions and NucleoSpin ${ }^{\circledR}$ Blood (Macherey-Nagel) for blood cell fractions, as per 200 the manufacturer's protocol. After extraction, the concentration of DNA each sample was 201 adjusted to $10 \mathrm{ng} / \mu \mathrm{L}$. The adjusted DNA was used to detect $h F S T$ and $C M V p$ gene fragments 202 using TaqMan qPCR.

203

204 3.2.3. Determination of specificity of the primers and TaqMan probe

205 The DNA samples from each specimen (whole blood, plasma, and blood cells) mentioned in

206 3.2.1. and 3.2.2. were pooled in a 1.5-mL tube. The pooled DNA was subjected to TaqMan 207 qPCR to detect the transgene fragment using the same primers and TaqMan probes as in 3.2.1. 208 and 3.2.2. After the TaqMan qPCR, the amplified product was collected in a 1.5-mL tube and 209 purified with NucleoSpin ${ }^{\circledR}$ Gel and PCR Clean-up (Macherey-Nagel). The purified and 210 amplified products were sequence by an external vendor (FASMAC, Kanagawa, Japan) using the 
211 Sanger Sequence Analysis Service. The obtained data were analyzed using CLC Sequence

212 Viewer ver. 8.0 (QIAGEN, Hilden, Germany) and BioEdit ver. 7.2.5 (developer: Tom Hall).

213

214 3.2.4. Long term detection of transgene fragments for intramuscular injection

215 Approximately, $50 \mu \mathrm{L}$ of whole blood was collected from the tail vein of six untreated mice aged

2167 weeks into a $1.5-\mathrm{mL}$ tube containing $150 \mu \mathrm{L}$ of EDTA-2Na/PBS mixture, which was used as a

217 pre-injection sample. Three days later, $2.5 \times 10^{12} \mathrm{vp} / \mathrm{mL}$ of $\mathrm{rAdV}<h F S T>$ was injected at $30 \mu \mathrm{L}$

218 into the tibialis anterior and at $50 \mu \mathrm{L}$ into the gastrocnemius. Thereafter, approximately $50 \mu \mathrm{L}$ of

219 whole blood was collected from the tail vein at 3,6 , and $12 \mathrm{~h}$, and $1,1.5,2,2.5,3,3.5,4$, and 5

220 days after injection.

221 Total DNA was extracted using a phenol/chloroform/isoamyl alcohol solution. After extraction,

222 the concentration of DNA in each sample was adjusted to 10 or $100 \mathrm{ng} / \mu \mathrm{L}$, and DNA was

223 subjected to TaqMan qPCR to detect $h F S T$ and CMVp gene fragments.

224

\section{4. Primer design and qPCR}

226 The sequences of primers and TaqMan probes are listed in Table S2. Primers and TaqMan

227 probes were synthesized by Integrated DNA Technologies (Coralville, IA, USA).

228 SYBR® FAST qPCR Master Mix (KK 4602; Kapa Biosystems, Wilmington, MA, USA) reagent 229 was used to perform SYBR-Green qPCR. The volume of the template solution, final

230 concentration of the primer, negative control, and experimental conditions were taken from a 231 previous study (Aoki et al., 2020).

232 PrimeTime Gene Expression Master Mix (Integrated DNA Technologies) reagent was used to 233 perform TaqMan-qPCR. The volume of template solution, final concentrations of primer and 234 probe, negative control, and experimental conditions were taken from a previous study (Aoki et 235 al., 2020). For standard curve for absolute quantification, $100 \mathrm{pg} / \mu \mathrm{L}$ Gen EZ ${ }^{\mathrm{TM}}$ ORF clone FST 236 in pcDNA3.1(+) was used. The coefficient of determination $\left(\mathrm{R}^{2}\right)$ of the calibration curve was set 237 to $\mathrm{R}^{2}>0.98$.

238 Both SYBR-Green qPCR and TaqMan-qPCR samples were run in duplicates, and QuantStudio 5 239 Real-Time PCR Systems (Thermo Fisher Scientific) were used.

240

\section{5. Statistical analysis}


242 GraphPad Prism version 7.04 (GraphPad, Inc., La Jolla, CA, USA) was used for statistical

243 analysis of the data. First, the Shapiro-Wilk normality test was performed on all data to check the

244 normality of the distributions. Then, data following a normal distribution were subjected to an

245 unpaired t-test or one-way analysis of variance test, and the Tukey-Kramer method was used for

246 the post hoc test. For non-normal distribution, Mann Whitney's U test and Benjamini, Krieger

247 and Yekutiel's two-stage method were used. p-values less than 0.05 were considered statistically

248 significant. Cell experiments data were indicated mean \pm SD. Animal experiments data were

249 indicated mean \pm SEM.

250

251

252 Results

$253 \boldsymbol{h F S T}$ gene and FST protein were overexpressed in $\mathrm{HuH} 7$ and $\mathrm{C} 2 \mathrm{C} 12$ cells

254 The expression of hFST and FST proteins in $\mathrm{HuH7}$ and $\mathrm{C} 2 \mathrm{C} 12$ cells significantly increased $(p<$ 255 0.001) in the group infected with $\mathrm{rAdV}<h F S T>$, compared with the group infected with PBS and 256 the group infected with rAdV $<m$ Cherry $>$ (Fig. 1A, C, Fig. 2A, C, D, Fig. S1A, Fig. S2A). The 257 number of hFST gene fragments and FST protein in the cell culture supernatant also increased 258 significantly $(p<0.001)$ (Fig. 1B, Fig. 2B, Fig. S1B, Fig. S2B), and the phosphorylation levels 259 of Akt and p70S6K significantly increased $(p<0.05)$ in $\mathrm{C} 2 \mathrm{C} 12$ cells (Fig. 2E, F).

260

261

262

Transgene fragments were detected for $\mathbf{4}$ days after intravenous injection

263

Compared with the pre-infection samples, hFST transgene fragments were detected until 2 days

264 $(p<0.05)$ and CMVp until 4 days $(p<0.05)$ after injection. In addition, both gene fragments

265

were the highest at $6 \mathrm{~h}$ after injection (Fig. 3).

\section{Transgene fragments were detected in each specimen of intravenous injection}

No transgene fragments were detected in any of the specimens in G1. In contrast, transgene

268 fragments, including of $\mathrm{hFST}$ and CMVp, were detected in both specimens in G2. Moreover, 269 when plasma and blood cell fractions were compared, transgene fragments were significantly

270 more localized in the plasma fraction $(p<0.05)$ (Fig. 4).

271

272

Primers and TaqMan probes were specific for the targeted transgene fragment 
273 The results of the amplification curve of TaqMan-qPCR confirmed that the $h F S T$ and $C M V p$ 274 gene fragments in whole blood, plasma, and blood cell samples were fully amplified. Sanger 275 sequencing showed that the sequences of all samples matched the reference sequences by more 276 than 90 bases (Fig. 5). This confirmed that amplification by the prepared primers and TaqMan 277 probe was specific.

278 279

Transgene fragments were detected until 3 days after intramuscular injection

281

282

283

284

285

286

287

288

289

290

291

292

293

294

295

296

297

298

299

300

301

302

303

Compared with the pre-infection serum, after intramuscular injection, $h F S T$ transgene fragments were detected in $10 \mathrm{ng} / \mu \mathrm{L}$ of injected DNA until $12 \mathrm{~h}(p<0.05)$ (Fig. 6A) and until 1 day $(p<$ 0.05) for $C M V p$ (Fig. 6C) and in $100 \mathrm{ng} / \mu \mathrm{L}$ of DNA until 2.5 days $(p<0.05$ ) for $h F S T$ (Fig. 6B) and until 3 days $(p<0.05)$ for $C M V p$ (Fig. 6D).

\section{Discussion}

In this study, we evaluated gene and protein expression and muscle protein synthesis signaling using $\mathrm{rAdV}<h F S T>$ and developed a detection method for gene doping by intravenous or intramuscular injection of $\mathrm{rAdV}<h F S T>$. In cell experiments, the $h F S T$ gene and FST protein expression in $\mathrm{HuH} 7$ and $\mathrm{C} 2 \mathrm{C} 12$ cells significantly increased after injecting $\mathrm{rAdV}<h F S T>$, suggesting that the $h F S T$ gene and FST protein expression in the liver or muscle might significantly increase after intravenous or intramuscular injection of $\mathrm{rAdV}<h F S T>$. In addition, because FST is secreted extracellularly, the significantly raised FST in the liver or muscle would be secreted into the blood or near the muscle, respectively. In addition, because the phosphorylation of Akt and p70S6K significantly increased in $\mathrm{C} 2 \mathrm{C} 12$ cells, it is expected that muscle synthesis would have been activated to induce muscle hypertrophy. The results of the cell experiments in this study suggested that intravenous or intramuscular injection of $\mathrm{rAdV}<h F S T>$ may be used to construct a gene-doping model.

We injected $\mathrm{rAdV}<h F S T>$ in mice intravenously or intramuscularly and then sampled blood over time to see how many days after injection it took for the transgene and $\mathrm{rAdV}$ vector fragments to disappear. The results showed that gene fragments could be detected up to 4 days after intravenous injection (Fig. 3) and up to 3 days after intramuscular injection (Fig. 6). To 
304 date, no study has attempted to detect the hFST gene fragment, and these results are considered

305 important findings for future research. However, further studies are needed to clarify the

306 detectable period of $\mathrm{rAdV}<h F S T>$ in humans. It has been reported that drug metabolism in small

307 experimental animals such as mice is about 10 times higher than that in humans (Kato, 1981),

308 and it is possible that the rate of metabolism of genes introduced by adenovirus vectors differs

309 between experimental animals and humans, but the extent of the difference is not yet clear. In

310 addition, compared with the amount of virus used in humans (Zhang et al., 2018), this study used

311 approximately 1/10 for intravenous injection and approximately 1/50 for intramuscular injection.

312 Considering the body weight ratio (2000:1) and circulating blood volume ratio (1500:1) of

313 humans (approximately $60 \mathrm{~kg}$ ) and mice (approximately $30 \mathrm{~g}$ ), a large amount of virus was

314 administered in this study.

315 Doping tests require highly sensitive detection methods. To determine localization of the

316 transgene fragments in whole blood, whole blood was collected and centrifuged into plasma and

317 blood cell fractions and analyzed to determine which fraction contained the highest number of

318 gene fragments. The $h F S T$ and $C M V p$ gene fragments were localized more in the plasma fraction

319 than in the blood cell fraction (Fig. 4). In contrast, in a previous study using rAdV, gene

320 fragments were more localized in the blood cell fraction than in the plasma fraction (Sugasawa et

321 al., 2019). This may be due to the time between injection and blood collection. Blood samples

322 were collected 5 days after injection in the previous study, whereas blood was sampled $6 \mathrm{~h}$ after

323 injection in this study. Because the injected rAdV vector was used as a viral solution, it is

324 presumed to have localized in the plasma immediately after injection. It infected red blood cells

325 or was phagocytosed by leukocytes over time. This is expected to lead to the development of

326 more sensitive detection methods by determining the exact time points at which gene fragments

327 localize predominantly in the plasma or in the blood cell fraction and which of the blood cell

328 components (erythrocytes, leukocytes) or the surfaces of blood cells have the most localization.

329 Doping tests should have a high specificity. Therefore, it is necessary to develop a method to

330 specifically detect target gene fragments. We used the Sanger sequencing method to verify the

331 specificity of the detection performed in this study. As a result, we were able to specifically

332 detect transgene fragments (Fig. 5). Furthermore, no non-specific amplification or contamination

333 was found in the control group or samples before rAdV vector injection. These results indicate

334 that the DNA extraction method, primer and TaqMan probe design, thermal cycling conditions, 
335 336

and reagents for TaqMan-qPCR in this study were accurate and optimal. Therefore, the protocol constructed in this study may be directly applicable to human specimens.

A limitation of this study was that we did not construct a gene doping model by intravenous or intramuscular injection of $\mathrm{rAdV}<h F S T>$. In other words, we were not able to establish a model that shows specific phenotypes of intravenous or intramuscular injection of $\operatorname{rAdV}<h F S T>$, such as changes in muscle strength, muscle wet weight, and muscle fiber type, owning to the increased levels of $h F S T$ gene and FST protein in the liver and muscle and activation of muscle synthesis. Therefore, it is necessary to establish a gene-doping model by intravenous or intramuscular injections in the future.

Research on methods for detecting gene doping has been conducted for only about 20 years, and it is still a developing research field with many unknowns. This study is a pioneering work in this field, and the results of this study may contribute to the development of detection methods for gene doping using $\mathrm{rAdV}<h F S T>$.

\section{Conclusions}

In this study, we aimed to develop a method to detect multiple transgene fragments as proof of gene doping using rAdV $<h F S T>$. Figure 7 presents a summary. This study showed that in the $\mathrm{rAdV}<h F S T>$ transgenic model, multiple transgene fragments could be detected using TaqManqPCR from as little as $50 \mu \mathrm{L}$ of whole blood, and each gene fragment was localized predominantly in the plasma. The new findings of this study may contribute to the development of detection methods for gene doping using $\mathrm{rAdV}<h F S T>$.

\section{Acknowledgements}

We would like to thank Editage (www.editage.com) for English language editing.

\section{Data Availability}

The following information was supplied regarding data availability: The raw data is available in the Supplementary Files

\section{References}


366 Gene therapy Clinical Trials. 2021. [(accessed on 5 March 2021)]. Available at:

367 www.abedia.com/wiley/.

368

369 Aoki K, Sugasawa T, Yanazawa K, Watanabe K, Takemasa T, Takeuchi Y, Aita Y, Yahagi N,

370 Yoshida Y, Kuji T, Sekine N, Takeuchi K, Ueda H, Kawakami Y, Takekoshi K. 2020. The

371 detection of trans gene fragments of hEPO in gene doping model mice by TaqMan qPCR assay.

372 PeerJ 8:e8595. doi:10.7717/peerj.8595.

373

374 Bodine SC, Stitt TN, Gonzalez M, Kline WO, Stover GL, Bauerlein R, Zlotchenko E,

375 Scrimgeour A, Lawrence JC, Glass DJ, Yancopoulos GD. 2001. Akt/mTOR pathway is a crucial 376 regulator of skeletal muscle hypertrophy and can prevent muscle atrophy in vivo. Nature Cell 377 Biology 3:1014-1019. doi:10.1038/ncb1101-1014.

378

379 DePaolo LV. 1997. Inhibins, activins, and follistatins: The saga continues. Proceedings of the 380 Society for Experimental Biology \& Medicine. Society for Experimental Biology \& Medicine 381 214:328-339. doi:10.3181/00379727-214-44100.

382 Duan D. 2018. Systemic AAV micro-dystrophin gene therapy for Duchenne muscular dystrophy. 383 Molecular Therapy: The Journal of the American Society of Gene Therapy 26:2337-2356.

384 doi:10.1016/j.ymthe.2018.07.011.

385 Patal K. 1998. Follistatin. The International Journal of Biochemistry \& Cell Biology 30:1087386 1093. doi:10.1016/s1357-2725(98)00064-8.

387

388 Hansen JS, Plomgaard P. 2016. Circulating follistatin in relation to energy metabolism.

389 Molecular \& Cellular Endocrinology 433:87-93. doi:10.1016/j.mce.2016.06.002.

390 High KA, Anguela XM. 2016. Adeno-associated viral vectors for the treatment of hemophilia.

391 Human Molecular Genetics 25:R36-41. doi:10.1093/hmg/ddv475.

392 Kato R. 1981. Species Differences in drug metabolism - overview from animals to man (author's 393 trans1). Jikken Dobutsu. Experimental Animals 30:507-517. PMID:ㄹ18948. 
395 Lee CS, Bishop ES, Zhang R, Yu X, Farina EM, Yan S, Zhao C, Zheng Z, Shu Y, Wu X, Lei J, 396 Li Y, Zhang W, Yang C, Wu K, Wu Y, Ho S, Athiviraham A, Lee MJ, Wolf JM, Reid RR, He 397 TC. 2017. Adenovirus-mediated gene delivery: Potential applications for gene and cell-based 398 therapies in the new era of personalized medicine. Genes \& Diseases 4:43-63.

399 doi:10.1016/j.gendis.2017.04.001.

400 Liang M. 2018. Oncorine, the World First Oncolytic Virus Medicine and its Update in China. 401 Current Cancer Drug Targets 18:171-176. doi:10.2174/1568009618666171129221503.

402 Naso MF, Tomkowicz B, Perry WL, Strohl WR. 2017. Adeno-associated virus (AAV) as a 403 vector for gene therapy. BioDrugs: Clinical Immunotherapeutics, Biopharmaceuticals \& Gene 404 Therapy 31:317-334. doi:10.1007/s40259-017-0234-5.

405 Press release Novartis. 2019. [(accessed on 10 March 2021)]. Available at:

406 https://www.novartis.com/news/media-releases/avexis-receives-fda-approval-zolgensma-first407 and-only-gene-therapy-pediatric-patients-spinal-muscular-atrophy-sma.

408 Press release Novartis. 2018. [(accessed on 10 March 2021)]. Available at:

409 https://www.novartis.com/news/media-releases/novartis-receives-european-commission410 approval-its-car-t-cell-therapy-kymriah-tisagenlecleucel.

411 Pharmaceuticals and Medical Devices Agency (PMDA). 2019. [(accessed on 10 March 2021)]. 412 Available at: https://www.pmda.go.jp/files/000231386.pdf.

413

414 Sugasawa T, Aoki K, Watanabe K, Yanazawa K, Natsume T, Takemasa T, Yamaguchi K, 415 Takeuchi Y, Aita Y, Yahagi N, Yoshida Y, Tokinoya K, Sekine N, Takeuchi K, Ueda H, 416 Kawakami Y, Shimizu S, Takekoshi K. 2019. Detection of transgenes in gene delivery model 417 mice by adenoviral vector using ddPCR. Genes 10:436. doi:10.3390/genes10060436.

418

419 The World Anti-Doping Agency (WADA). 2021. World anti-doping code with international 420 standard, prohibited list. Montreal, QC, Canada: WADA.

421

422 The World Anti-Doping Agency (WADA). 2015. Anti-doping textbook. Montreal, QC, Canada: 423 WADA. 
424 http://antidopinglearninghub.org/sites/default/files/supporting-material/Anti-

425 Doping\%20Textbook\%20-\%202015\%20Code.pdf

426

427 The World Anti-Doping Agency (WADA). 2021. Laboratory Guidelines, Gene Doping

428 Detection based Polymerase Chain Reaction (PCR). Montreal, QC, Canada: WADA.

429 https://www.wada-

430 ama.org/sites/default/files/resources/files/wada guidelines for gene doping per test v1 jan 2021

431 eng.pdf

432

433 The World Anti-Doping Agency (WADA). 2020. World anti-doping code with international

434 standard, prohibited list. Montreal, QC, Canada: WADA.

435

436 Wang F, Qin Z, Lu H, He S, Luo J, Jin C, Song X. 2019. Clinical translation of gene medicine.

437 The Journal of Gene Medicine 21:e3108. doi:10.1002/jgm.3108.

438

439 Wold WS, Toth K. 2013. Adenovirus Vectors for Gene Therapy, vaccination and cancer gene

440 therapy. Current Gene Therapy 13:421-433. doi:10.2174/1566523213666131125095046.

441 Xia Y, Du Z, Wang X, Li X. 2018. Treatment of uterine sarcoma with rAd-p53 (Gendicine)

442 followed by chemotherapy: Clinical study of TP53 gene therapy. Human Gene Therapy 29:242-

443 250. doi:10.1089/hum.2017.206.

444

445 Zhang WW, Li L, Li D, Liu J, Li X, Li W, Xu X, Zhang MJ, Chandler LA, Lin H, Hu A, Xu W, 446 Lam DM. 2018. The first approved gene therapy product for cancer ad- p53 (Gendicine): 12

447 years in the clinic. Human Gene Therapy 29:160-179. doi:10.1089/hum.2017.218.

448

449 


\section{Table $\mathbf{1}$ (on next page)}

Table 1. Types and relative numbers of the top seven clinically approved vectors used in gene therapy. 


\begin{tabular}{ccc}
\hline \multirow{2}{*}{ Vector } & \multicolumn{2}{c}{ Gene Therapy Clinical Trials } \\
& Number & $\%$ \\
\hline \hline Adenovirus & 573 & 17.5 \\
Retrovirus & 536 & 16.4 \\
Naked/Plasmid DNA & 482 & 14.7 \\
Lentivirus & 331 & 10.1 \\
Adeno-associated virus & 263 & 8.0 \\
Vaccinia virus & 197 & 6.0 \\
Lipofection & 125 & 3.8 \\
Others & 673 & 23.5 \\
Total & 3180 & 100 \\
\hline
\end{tabular}


Figure 1

Figure 1. Evaluation of gene and protein expression by $r A d V<h F S T>$ in $\mathrm{HuH7}$.

A; hFST gene expression. B; Detection of hFST gene fragments in culture supernatant. C; FST protein expression in cells. To confirm the rAdV was completely working, cell experiments were conducted. HuH7 cell line established from a highly differentiated human hepatocellular carcinoma. hFST gene and protein were overexpressed in hFST group. hFST gene fragment was detected from cell supernatant in hFST group. Data are means \pm SD.*** $p<0.001$.
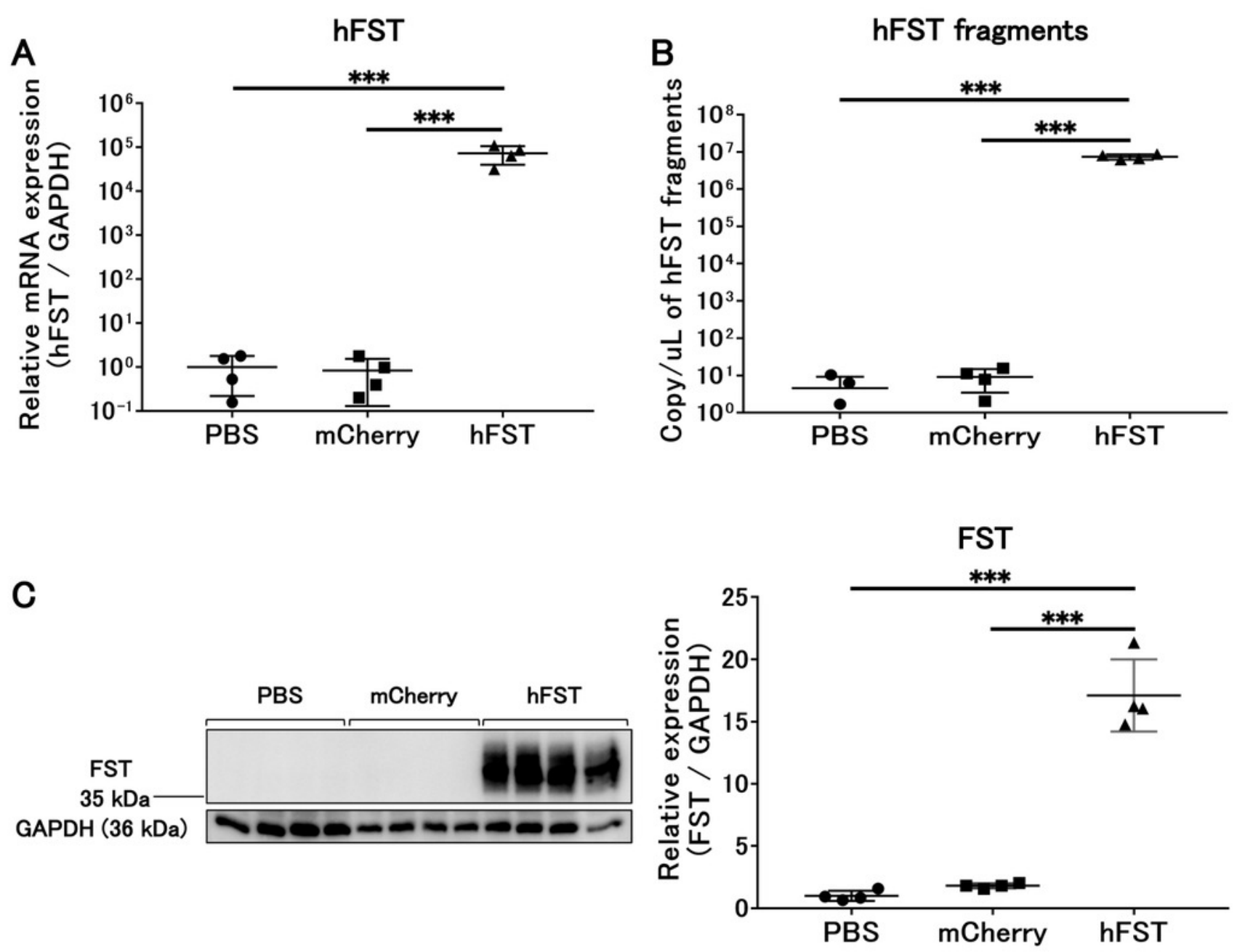


\section{Figure 2}

Evaluation of gene and protein expression and muscle protein synthesis signaling activity by $\mathrm{rAdV}<\mathrm{hFST}>$ in $\mathrm{C} 2 \mathrm{C} 12$.

A; hFST gene expression. B; Detection of hFST gene fragments in culture supernatant. C; FST protein expression detected by western blot. D; FST protein expression detected by immunofluorescence. E; Akt phosphorylation level. F; p70S6K phosphorylation level. To confirm the rAdV was completely working and muscle synthesis signals were activated, cell experiments were conducted. $\mathrm{C} 2 \mathrm{C} 12$ is an immortalized mouse myoblast cell line. hFST gene and protein were overexpressed in hFST group. hFST gene fragment was detected from cell supernatant in hFST group. The phosphorylation levels of Akt and p70S6K significantly increased in hFST group. Data are means \pm SD. $* p<0.05$ and $* * * p<0.001$. 

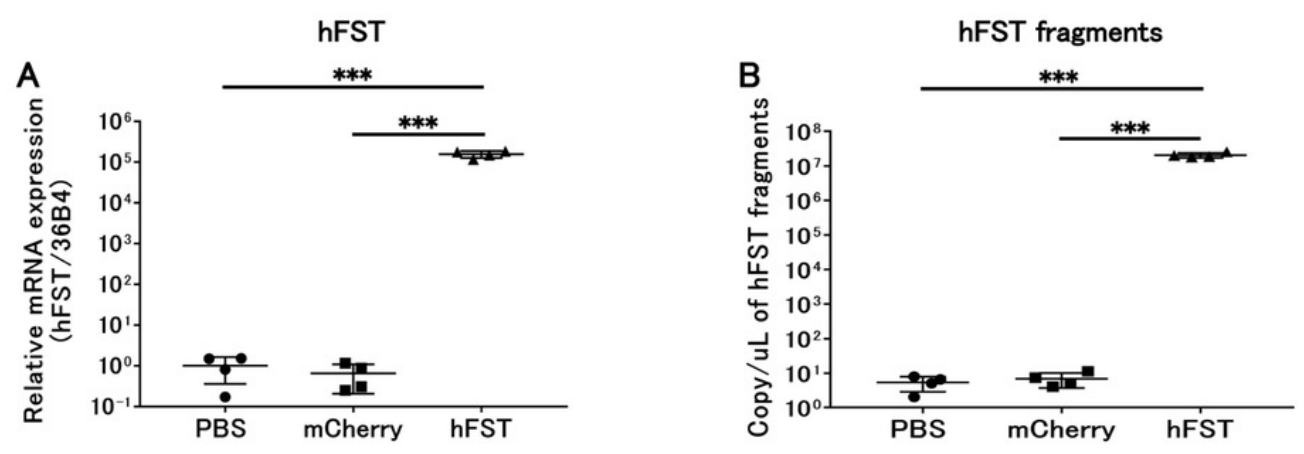

C

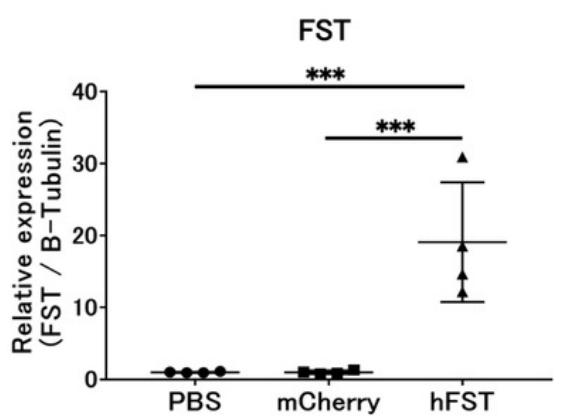

D
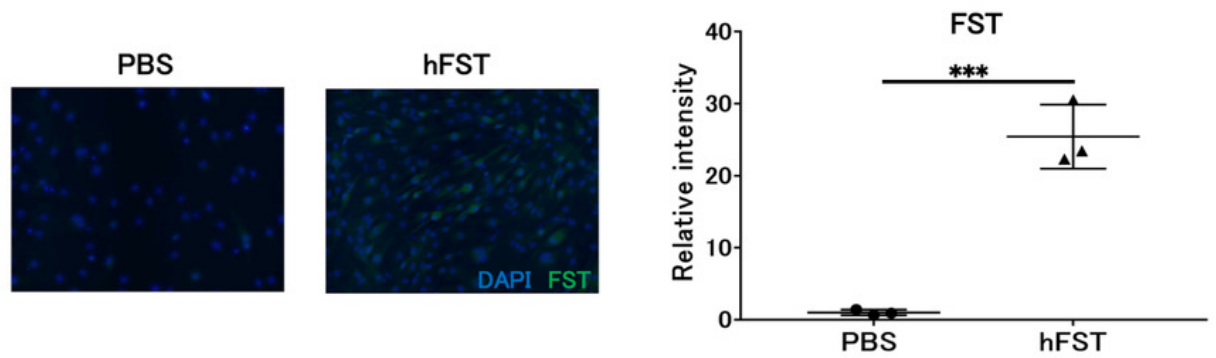

E

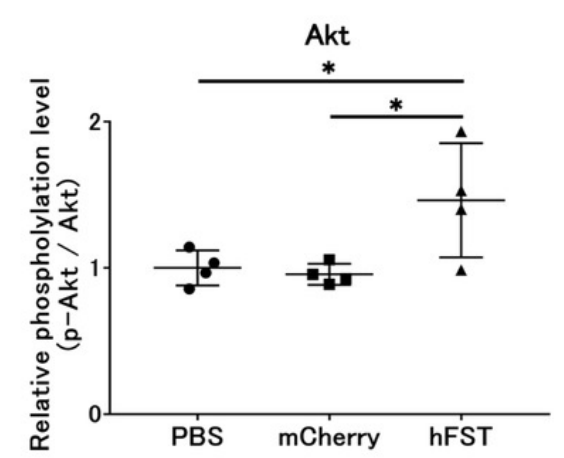

F
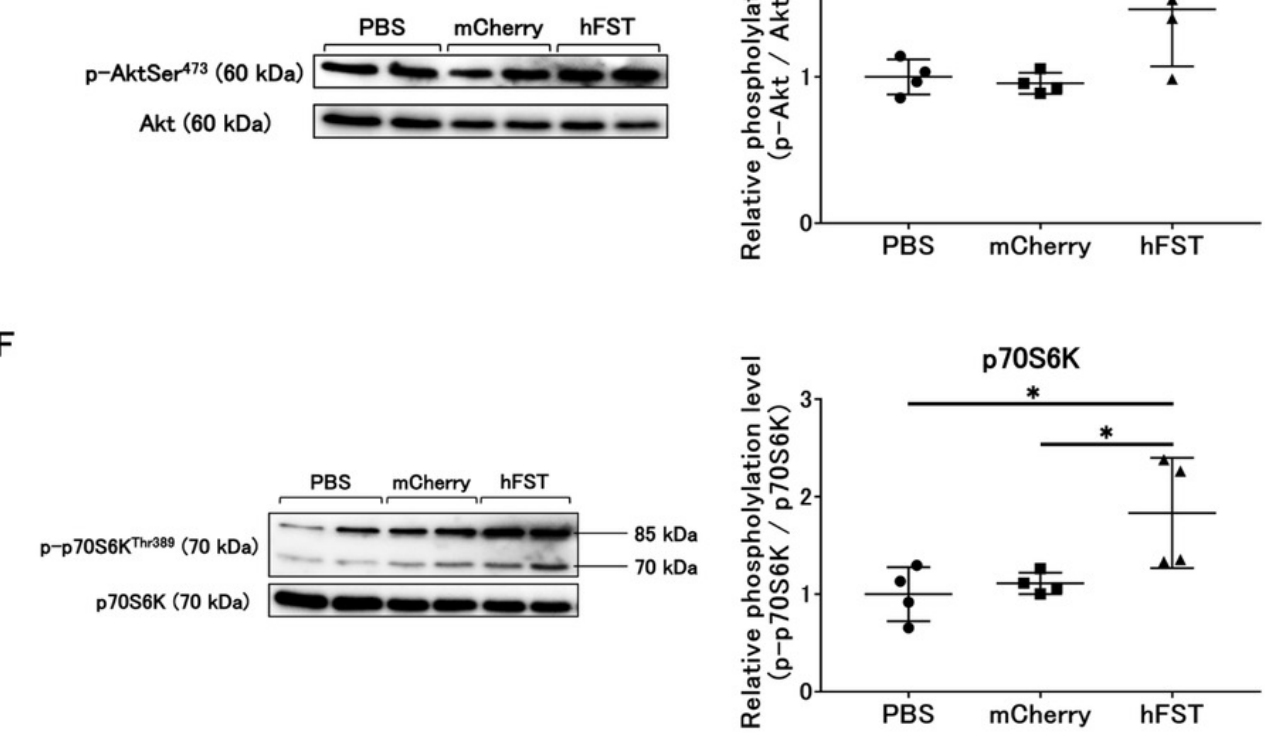


\section{Figure 3}

Figure 3. Long-term detection of transgene fragments by intravenous injection.

A; Detection of hFST gene fragments. B; Detection of CMVp gene fragments. To confirm the detection period of the transgene fragments, animal experiments were conducted. rAdV was injected through the orbital venous plexus. hFST transgene fragments were detected until 2 days and CMVp until 4 days after injection. ND; not detected. Data are means $\pm S E M . * p<$ 0.05 , ** $p<0.01$, and ${ }^{* * *} p<0.001$ vs. the pre-values before the injection.
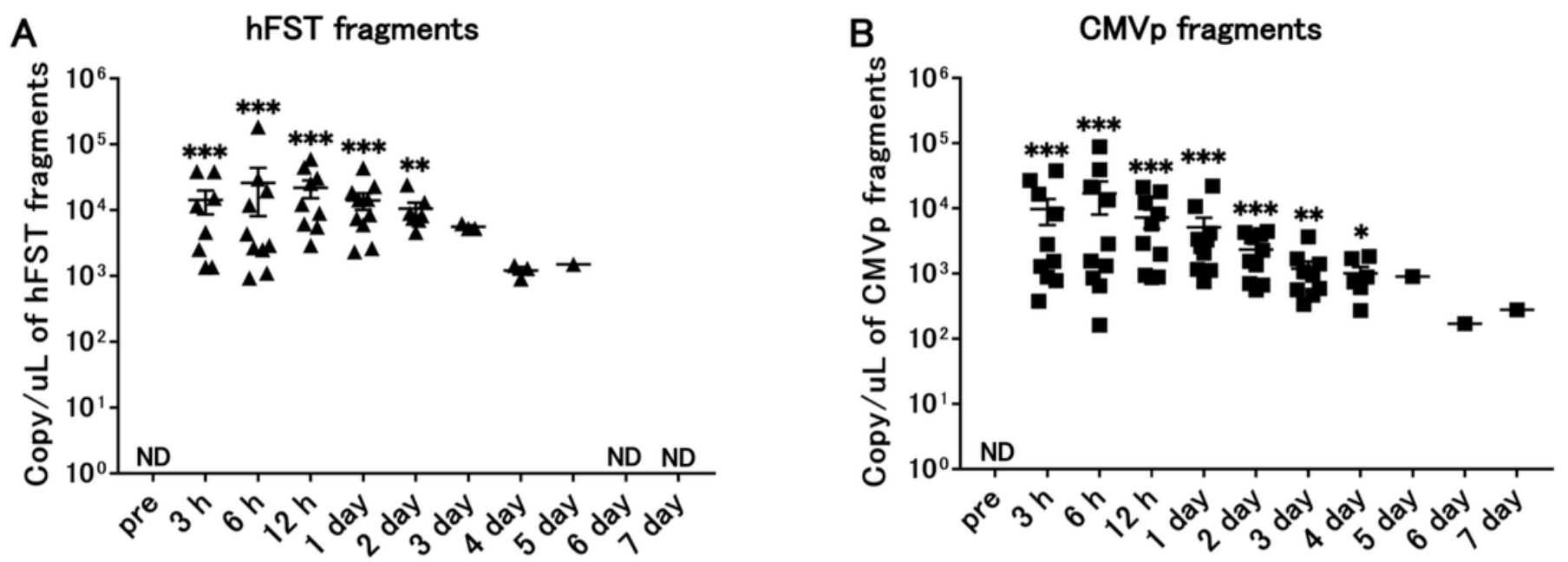


\section{Figure 4}

Figure 4. Detection of transgene fragments from each sample by intravenous injection.

A; Detection of hFST gene fragments in blood-cell and plasma fractions. B; Detection of CMVp gene fragments in blood-cell and plasma fractions. To confirm the localization of the transgene fragment in the blood, animal experiments were conducted. hFST and CMVp were detected in plasma and blood cell fractions. Moreover, when plasma and blood cell fractions were compared, transgene fragments were significantly more localized in the plasma fraction. Data are means \pm SEM. $* p<0.05$.

A

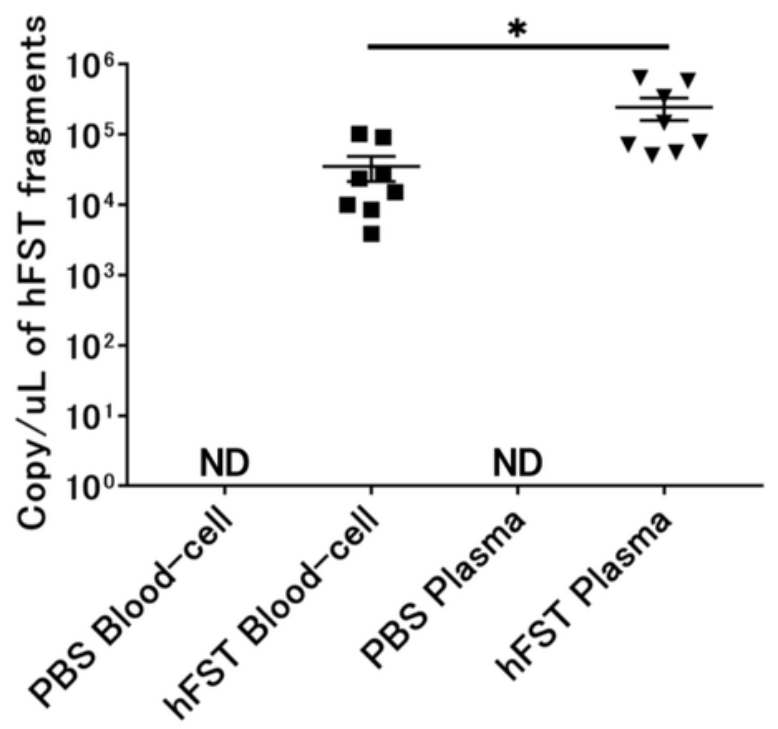

B $\quad$ CMVp fragments

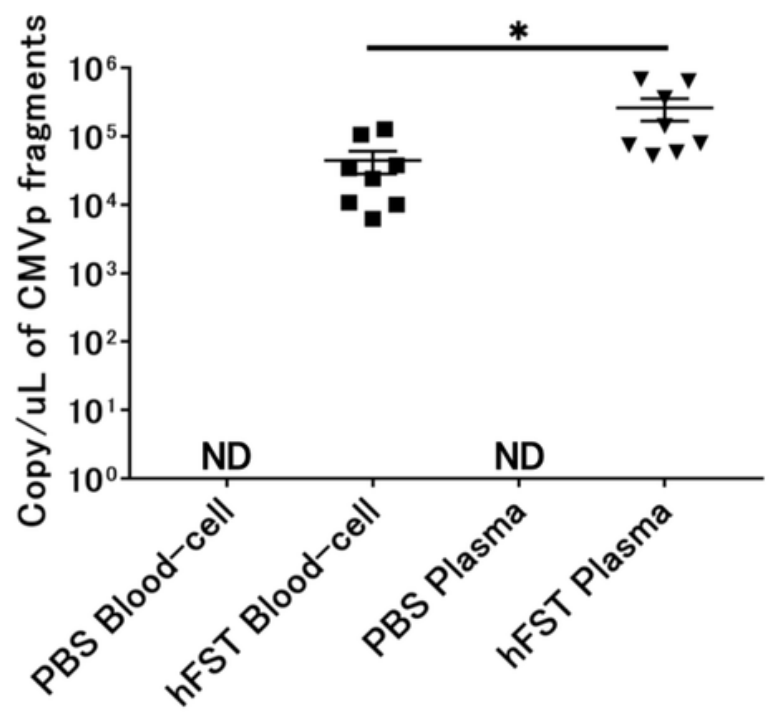




\section{Figure 5}

Figure 5. Evaluation of the specificity of primers and TaqMan probes.

A; Sequence of about 40 base pairs obtained from the amplification curve of the hFST gene fragments by TaqMan-qPCR and the waveform of the Sanger sequencing method. B;

Sequence of about 40 base pairs obtained from the amplification curve of the CMVp gene fragment by TaqMan-qPCR and the waveform of the Sanger sequencing method. PC means positive control (100 pg/uL pFST), WB means whole-blood DNA, B means blood-cell-fraction DNA, and P means plasma cfDNA. To confirm the specificity of the primers and TaqMan probe, Sanger Sequence Analysis was conducted. Primers and TaqMan probes were specific for the targeted transgene fragments. 
A

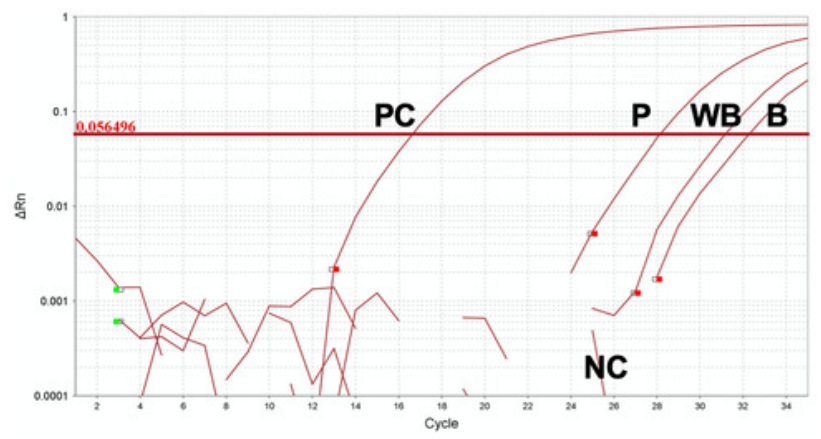

Reference Sequence (40 bp) GCCTGCTGGGCAGATCTATTGGATTAGCCTATGAGGGAAA GCCT GCT GGGCAGAT CTAT I G GAT TA GCCTAT GAGGGAAA PC

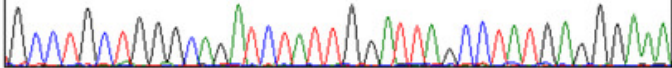
G C C T GC T G G G CAG AT C TAT T G GAT TA GCC TAT GAG G GAAA WB

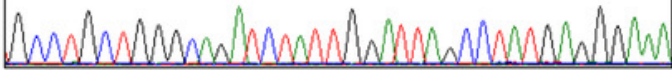
GCCTGCT GGGCAGAT CTAT T G GAT TAGCCTAT GAGGGAAA B

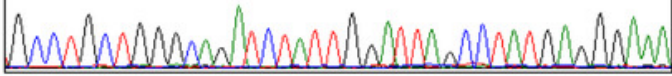
GCCTGCT GGGCAGATC TAT T G GAT TA GCCTAT GAGGGAAA $\mathbf{P}$

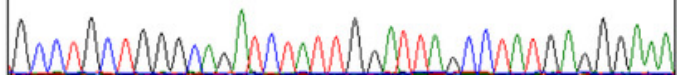

B

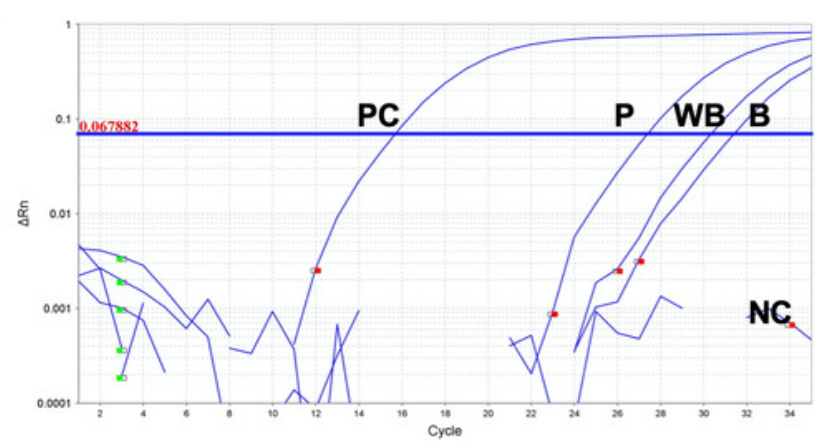

Reference Sequence (40 bp) ATGACCTTATGGGACTTTCCTACTTGGCAGTACATCTACG

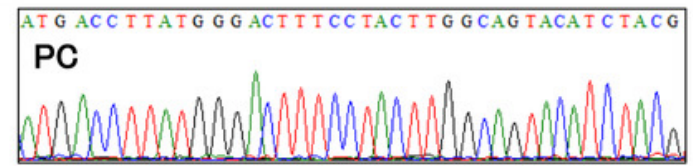
AT GACC T TATG GGACT T TCTAC T T G GCAGTACATCTACG WB

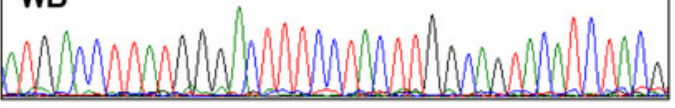
A I G ACC I TAT G G G AC T I I CC TAC T I G G CA G TACA T C TACG B

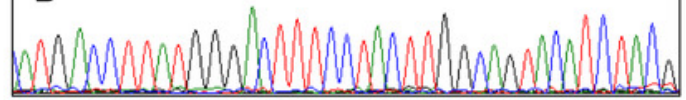
AI G ACC I TAT G G G AC I I I CC TAC I T G G CAG TACA I C TACG $\mathbf{P}$

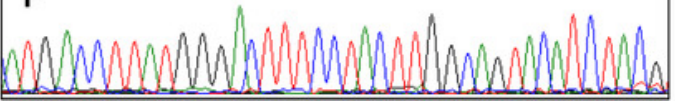




\section{Figure 6}

Figure 6. Long-term detection of transgene fragments for intramuscular injection.

A; Detection of hFST gene fragment using a sample with DNA concentration of $10 \mathrm{ng} / \mathrm{uL}$; $\mathrm{B}$; Detection of hFST gene fragment using a sample with DNA concentration of $100 \mathrm{ng} / \mathrm{uL}$; C; Detection of CMVp gene fragment using a sample with DNA concentration of $10 \mathrm{ng} / \mathrm{uL}$; ; Detection of CMVp gene fragment using a sample with DNA concentration of $100 \mathrm{ng} / \mathrm{uL}$. D; detection of CMVp gene fragments using a sample with a DNA concentration of $100 \mathrm{ng} / \mathrm{uL}$. To confirm the detection period of the transgene fragments, animal experiments were conducted. rAdV was injected into the tibialis anterior and gastrocnemius. hFST transgene fragments were detected upon 2.5 days and upon 3 days for CMVp. ND; not detected. Data are means \pm SEM. $* p<0.05, * * p<0.01$, and $* * * p<0.001$ vs. the pre-values before the injection. 

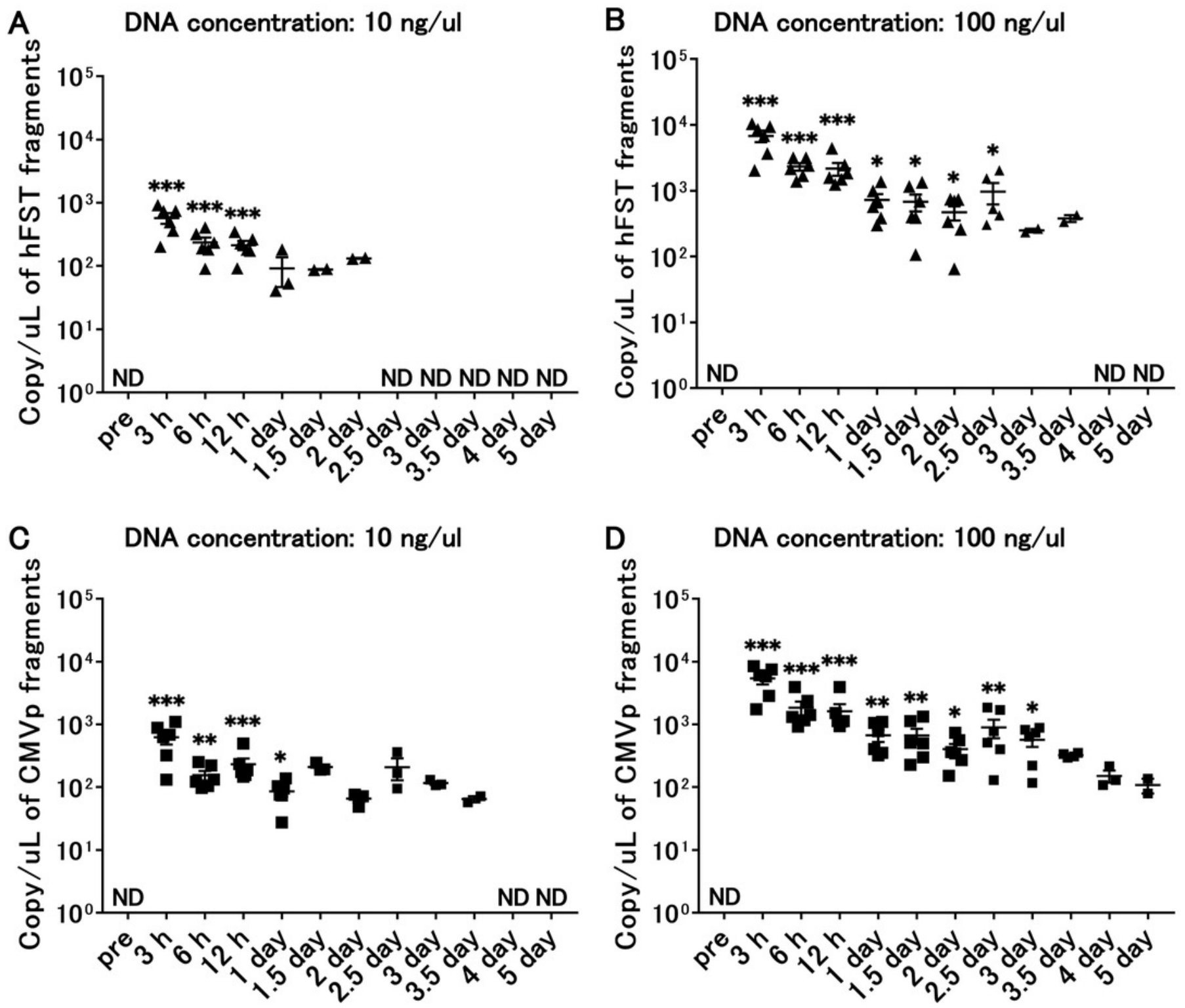
Figure 7

Figure.7. Summary of this study

\section{Cell experiments}

rAdV vector

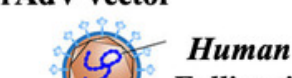

y

Follistatin

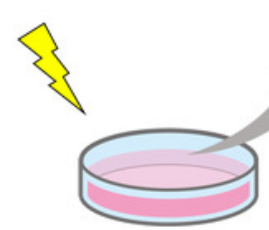

HuH7
rAdV vector

hFST gene

FST protein
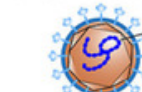

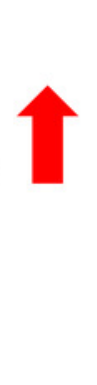

Human

Follistatin

hFST gene

FST protein

Akt phosphorylation level p70S6K phosphorylation level
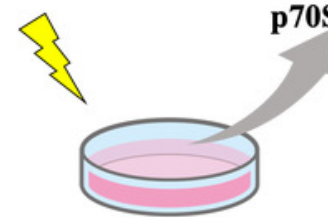

rAdV $<h F S T>$ induces overexpression of hFST gene and FST protein, and increased phosphorylation levels of Akt and p70S6K in C2C12.

\section{Animal experiments}

rAdV vector

intravenous

or

Human intramuscular Follistatin

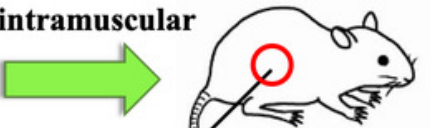

Blood collection from abdominal vena cava

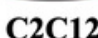

\begin{tabular}{|c|c|c|}
\hline rAdV $<\boldsymbol{h F S T}>$ & hFST gene fragment & CMVp gene fragment \\
\hline intravenous & $\mathbf{2}$ days & $\mathbf{4}$ days \\
\hline intramuscular & 2.5 days & $\mathbf{3}$ days \\
\hline
\end{tabular}

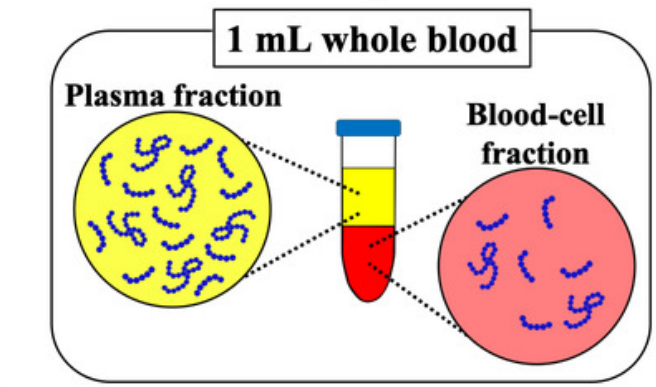

Blood collection from tail vein

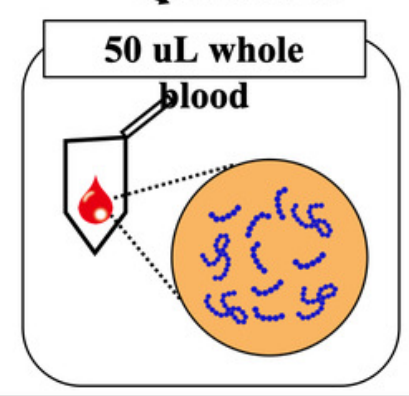

The combination of plasma cfDNA or one drop of whole blood with TaqMan qPCR may contribute to the development of a detection method for gene doping using $\operatorname{rAdV}<\boldsymbol{h F S T}\rangle$. 